\title{
The importance of leadership art in management
}

\author{
Yanzhe ZHENG \\ SHENZHEN ET TECHNOLOGY CO., LTD, Shenzhen, Guangdong, China
}

\begin{abstract}
- the leadership of the art is the comprehensive reflection of the individual quality of the leader, it is individual from person to person. Leadership art is the creative and the validity of the method which is demonstrated in the way of leadership. On the one hand, it is the creative and free creativity of the truth, goodness and beauty. "True" is the master rule, in law creation sublimation, sublimation to the artistic realm; "good" is to conform to the political idea; "the United States" refers to the leadership of people happy, comfortable. On the other hand, it is effective and the leadership practice is the only standard to examine the art of leadership.
\end{abstract}

Keywords - Leadership Art, Administration, Communicate, Leadership

浅谈领导艺术在管理中的重要性

\author{
郑彦哲 \\ 深圳市尔泰科技有限公司, 深圳, 广东, 中国
}

\begin{abstract}
摘 要 领导艺术是领导者个人素质的综合反映, 是因人而异的。领导艺术是指在领导的方式方法上表现出的创造性和有效性。 一方面是创造, 是真善美在领导活动中的自由创造性。“真” 是把握规律, 在规律中创造升华, 升华到艺术境界; “善” 就是要符合政 治理念; “美” 是指领导使人愉悦、舒畅。另一方面是有效性, 领导实践活动是检验领导艺术的唯一标准。
\end{abstract}

关键词 领导艺术, 管理, 沟通, 领导力

所谓领导艺术, 就是领导者在其知识、经验、才能和 气质等因素的基础上形成的, 巧妙地运用各种领导条件、 领导原则和领导方法的基本技能。领导人有两个工作的 内容, 一个是 “人”, 一个是 “事”, 有两个原因使得 人比事重要。第一, 事是人做的。人的问题不解决, 事的问题会一再出现。第二, 所有的资源都要通过人 来发挥作用。但是人又是最复杂、最难领导和管理的。 人的复杂表现在他不是理性的, 而是感性的; 不是静 止的, 而是变动的。随着经济领域中 “智慧经济” 的 比重不断增加, 市场竞争变幻莫测, 人的位置越来越 重要。可以说, 领导人成就的大小, 最终取决于他整 合人力资源能力的大小。

下面就从领导艺术的几种表现形式来阐述领导艺 术在管理中的重要性:

\section{1. 用人和用权艺术}

领导者的用人艺术, 具体来说, 可以分为择人艺术和 人才管理两个方面, 也就是 “知人善任” 了。要 “知人”,
首先要对所需、所用之人有一个较全面的了解。在 “知人” 的基础上才有可能 “择” 合适的人才, 在某种程度上讲, “知人” 即为 “择人” 了, 因而 “知人” 就成为领导者用 人的第一要素与前提。当然, “知人” 识才是为了 “善任”, 通过 “善任” 人才来获得企业持续的竞争力才是用人艺术 最终落脚点。要用好人才, 就必须 “择人任势”。一个人, 不可能具备种种才能, 胜任一切岗位, 某一特定人才总有 最适合于他的位子。这就需要管理者在 “知人” 的基础上, 对人才的使用上给予恰当安排, 形成人员配置的最佳组合 机构, 达成最佳组合, 此即领导者的人才管理艺术。

首先, 领导者可以不知道下属的短处, 但不能不知道 他的长处。只关注人才的短处, 是很难发现企业所需的合 适人才的, 正所谓 “尺有所短, 寸有所长” 也即这个道理 了, 领导者必须将其发掘出来并加上合理的运用, 才算是 艺术地 “知人” 和 “择人”。其次, 伯乐虽然在 “择人” 的方面有很重要的作用, 但毕竟个人精力有限, 伯乐再尽 心尽力, 其相中的人也是有限的。因此, 企业更需要的是 
制定出人才的机制。企业领导者建立和完善一个公开、公 平、公正的 “择人” 机制, 让一匹匹千里马自己亮相也是 非常重要的。挑选最好的人才是领导者最重要的职责, 领 导者的工作, 就是每天把全世界各地最优秀的人才延揽过 来。

\section{1 识人先识己}

(1) 克服求同弃异的排他心理。只喜欢与自己兴趣爱 好, 志向相同的人, 无形之中, 在对待人才的使用上, 给 领导者自己拟下一个圈子, 这样既阻碍自己的视野, 同时 容易形成一切以领导者兴趣, 爱好为主, 以工作为人人的 现象，不利于领导者的工作的顺利开展。

（2）要克服狭险的䃚妒心理。害怕 “功高震主”，对 有才能的人见贤思妒，视而不见，这是领导者对自己驾驭 能力信心不足的一种表现，一方面不利于领导者自己能力 的提高, 另一方面不能人尽其用。

（3）工作出发, 增加相容性, 贵和不贵同。收集人才 一切应从工作的角度出发, 由于各人水平, 阅历年龄所教 育程度不同等原因, 其性格、爱好, 看问题的角度及工作 方式、方法等均会多样性, 在这一点上, 领导者应有宽广 的胸怀和客观的态度, 只要在原则问题上, 工作目标上一 致，就不应排斥对人才的使用。

\section{2 识人要识心}

考核领导者事业的成败，首先用事实来检查他所用之 人是唯贤、唯亲, 是人才、奴才, 还是庸才, 就可以推断 领导者的贤或愚, 或别有所投, 善于选贤任能, 为人才登 上最能发挥作用的岗位, 这才是一个领导人应有的气魄和 品质, 也最衡量领导者一个重要标志。

在人才选拔上任人唯贤。无论是古代还是现代, 无论 是东方还是西方国家, 都有过行政组织领导者任人唯亲, 导致行政组织内部近亲繁殖, 帮派林立, 最终导致行政系 统内部严重危机以致灭户的结果。因此作为一个现代科学 的领导者, 必须摒弃私心, 惜才爱才 “亲贤臣而远小人” 唯才是举, 选择出最优最佳的人才, 使下属的作用发挥更 充分。

\section{3 识人要领}

事在人为: 做事之前要先读懂人。成大事者先识人; 人际交往先读人; 努力摘掉有色眼镜; 见树木更要见森林。 人为主：从第一印象看人; 从笑容来断定你的心; 以发型来
判断对方; 从帽子的选择上来判断他人; 由眼镜观察对方; 从领带断定对方的生格。相由心生: 从性格上洞察人心 “善 任”一领导者的人才管理艺术。天下无无用之才。

1.4 要有正确态度

作为一个优秀的领导者，用人所长应做到：

（1）善于发现，不能 “一叶障目”一个下属可能有 许多缺点, 但有一项优势, 而且适应工作的一方面, 领导 者应当善于利用下属的优势, 并创造机会使其充分发挥, 以弥补其它方面的不足。

（2）善于运用，充分发挥人才特点 每个人在社会中 生活都需要有一个最佳位置, 但往往不能一发到位, 这就 需要领导者帮助他们找到最佳位置。

另外, 在人才的配备上, 要依据人的不同特性, 合理 配备, 这样才能做到人尽其用, 用其所长, 达到最佳组合, 要根据不同职能要求, 安排不同类型的人才, 同时, 不同 个性的人才根据个性的弱点配备能克服这种弱点的搭当, 真正做到优势互补, 同时能把个性特点对组织的损害程度 降低, 真正做到避其所短, 扬其所长。

\section{2. 决策艺术}

决策是领导工作的中心环节。决策是从多种方案中做 出的选择或决定, 也可以说是多方案择优。通常讲的领导 “拍板”，指的就是决策，但绝不能把决策仅仅理解为一 瞬间的 “拍板”，它包括决策前的提出问题、搜集资料、 预测未来、确定目标、拟订方案、分析估计和优选以及实 施中的控制和反馈、必要的追踪等全过程。决策是人类社 会确定方针、策略的大计活动。它涉及到人的生活的各个 领域, 人和集体的各种行动都受决策的支配, 它是有意识 地指导人们的行动走向未来预定的目标。

决策的主体是领导, 看一个领导的水平高下最主要的 是看决策。所以每个领导都必须精心制作决策, 科学执行 决策。领导决策程序, 即领导根据决策过程的内在规律性 所决定的既相互独立又前后联系的基本步骤来进行决策。 各种决策都具有共性, 一些基本的步骤是任何决策都必须 遵循的, 违背了这些基本步骤, 会使领导决策失去其科学 性, 甚至会导致领导决策的失败。一个完整的合理的决策 过程应该包括四个步骤。

其一, 界定问题, 确定决策目标。确立决策目标是领 导决策程序的第一基本步骤。目标直接关系决策的方向, 而问题是确立决策目标的需要和可能。因此, 领导只有找 准了问题, 分析问题发生的原因, 才能有针对性地确定决 
策目标，提出解决问题、实现决策目标的措施或方法。发 现决策问题是领导的重要职责。明确了决策问题就可以确 立决策目标。目标既是领导决策的出发点, 也是领导决策 的归宿。因此, 确立决策目标是领导决策的重要内容和前 提。确立决策目标时应注意几点：（1）目标要明确具体; （2）目标要切合实际; （3）如果是多目标决策，决策目 标应有主次之分; (4) 目标要有价值准则。

其二, 拟定各种可能的备选方案。拟定备选方案是领 导为实现特定的决策目标所选择的途径和手段。拟定方案 应遵循两个原则：（1）多样性原则。拟定方案要尽可能的 多, 尽量把各方面可能性都考虑在内。（2）独立性原则。 虽然拟定备选方案要求多样性、全面性, 但各个方案之间 的关系是一种相互排斥的关系, 即每个方案本身都是独立 的, 各个方案之间应有原则的差异。否则就无法划清界限 和比较优势, 也无法挑出最佳方案。方案拟定一般要经过 两个步骤：第一, 轮廓设想。第二, 细部设计。

其三，从各种备选方案中选出最合适的方案。在拟定 的各个备选方案中进行评价、比较和选择。这是领导决策 程序中最重要、最关键的步骤。方案评选应遵循几个标准:

（1）价值标准, 主要指决策方案的作用、效果、益处和意 义等, 主要是经济价值、社会价值、学术价值; (2) 最优 标准与满意标准, 最优指投入和付出的代价最小、获得的 效益最大;（3）局部服从全局标准;（4）期望值标准, 就是哪个方案的期望值最优，就选哪个方案。

其四, 决策的执行。领导首先要组织有关人员制定决 策方案的具体实施计划、措施和步骤。然后根据计划逐步 付诸实施。在制定方案的实施计划中，领导要充分考虑到 决策方案在实施中可能会出现什么问题, 以及这些问题出 现后会产生什么样的影响和危害。为此, 就需要准备一些 防范措施和应急措施, 以减少那些问题出现的可能性和危 害性。另外，由于决策是一个动态的、依赖于时空变量的 复杂过程, 有时可能会出现决策方案不同程度地偏离目标 的情况，所以，在决策方案实施、执行中，特别是如果主 客观条件发生重大变化以至必须重新确定决策目标时, 就 需要进行追踪决策。

领导要善于决策, 不善于决策的领导是低效能的领导。 领导的决策合理、及时、有艺术，群众拥护，心情舒畅， 就会政令畅通。领导的决策艺术主要表现在两个方面：第 一，下放决策权。第二，容缓决策时间。这里有个词 “合 理地” ：对于某个特定的、迫在眉睫的决策，来定义 “合 理地” 的范围, 是一门需要大量实践和技术的艺术。

\section{3. 人际关系的艺术}

人际关系是人们在活动过程中直接的心理关系, 它是 人们社会交往的基础, 也是人们日常生活、社会活动所不 可缺少的。人际关系是我们生活中的一个重要组成部分。 一些伟人也从自己的成功经验中总结出: 人际沟通能力是 非常重要的能力。比如: 罗斯福说 : “成功的公式中, 最 重要的一项能力是与人相处的能力。” 还有一位哲人也说 过， “没有交际能力的人，就像陆地上的船，永远到不了 人生的大海”。可见, 巧妙地进行人际沟通, 在工作、生 活中都非常重要。它对一个企业发展的所起的作用也是不 言而喻的。

首先, 巧妙的人际沟通可以提高企业成员的满意度。 巧妙的人际沟通容易使成员感到愉快、亲切、随和。这样 的感觉能制造出轻松愉悦的工作氛围, 能给员工带来积极、 健康、饱满的精神状态, 从而使成员对工作满意, 能更好 地投入到各项工作中，不断地为企业发展做出贡献。

第二, 巧妙的人际沟通可以增强企业成员的凝聚力。 巧妙地进行人际沟通能释放和缓解压力, 能使大事化小, 小事化了，从而起到维护人际关系的作用。企业中成员之 间人际关系和谐，大家团结一心，成员之间不会为一些鸡 毛算屁的事发生争执, 成员把心思花在如何完成企业目标 上，这样企业成员的凝聚力就会增强。

第三，巧妙的人际沟通可以提高企业成员的工作效率。 成员工作效率的高低直接影响着企业效益的好坏。虽然, 影响成员工作效率的原因是多方面的, 但是成员之间人际 沟通艺术是其中一个重要的因素。巧妙的人际沟通是创造 良好的人际关系的前提, 良好人际关系可以激发组织成员 的责任感和荣誉感, 可以激励成员的工作激情, 形成和谐 的气氛，从而提高成员的工作积极性、主动性和创造性, 进而提高工作效率。

人与人之间的沟通与交往, 不仅是一种出自本能的需 要, 也是适应社会发展、个人进步的必不可少的途径。交 往作为人类生存与发展赖以继续的一种行为模式, 在人类 社会的发展历程中扮演着重要角色。现今社会, 具有较强 的交往能力, 是个人立足于社会并求得发展的重要条件。 交往使我们彼此了解, 交往使我们互通有无, 交往使我们 化干戈为玉帛。今天我们无论是人际交往的广度还是深度, 都达到了一个前所未有的程度。

综上所述, 领导力是一门综合的艺术。它不仅仅 包含了各种具体的管理技能和管理方法, 也囊括了前瞻与 规划、沟通与协调、真诚与均衡等更诸多要素。要想成为 一名符合 21 世纪产业发展要求的管理者, 要想具备一名卓 
越管理者应有的基本素质，我们就必须在宏观决策、管理 行为、个人品质等不同层面认真学习、体会并实践 21 世纪 的领导艺术。当今市场经济社会，国家、企业及个人面临 的竞争异常激烈, 作为各级领导或管理者, 除了要认真研 究领导科学, 大力推行 标准化、规范化和科学化管理外, 更要进一步提高领导艺术, 创新工作方式方法。只有这样, 才能带领团队有效抵御化解各种风险, 保障其始终充满活 力, 不断占领制胜的高地。

\section{参考文献(References)}

[1] Liang Zhongming. Leadership theory, Peking University press, 2007.4

[2] Jiang Fakui Liu Yinhua. Leadership science, Dongbei University of Finance and Economics press, 2011.2

[3] Li Dian. Business leadership art discussion, modern business and trade industry, 2008. 9

[4] Song Yingtao. On the enterprise leadership and enterprise core competitiveness , China business, 201111 period 